\title{
Synthesis of an Enzyme-Mediated Reversible Crosslinked Hydrogel for Cell Culture
}

\author{
Kelti A. Smith, ${ }^{1,2,3^{*}}$ Mickael Dang,,$^{1,2,3^{*}}$ Alexander E. G. Baker, ${ }^{1,2,3}$ Tobias Fuehrmann, ${ }^{1,2}$ Ana \\ Fokina, ${ }^{1,2}$ and Molly S. Shoichet ${ }^{1,2,3 \dagger}$ \\ ${ }^{1}$ Department of Chemical Engineering and Applied Chemistry, University of Toronto, 200 College St, Toronto, \\ ON M5S 3E5, Canada \\ ${ }^{2}$ Donnelly Centre, University of Toronto, 160 College St, Toronto, ON M5S 3E1, Canada \\ ${ }^{3}$ Institute of Biomedical Engineering, University of Toronto, 160 College St, Toronto, ON M5S 3E1, Canada \\ * These authors contributed equally to this work. \\ + To whom correspondence should be addressed: molly.shoichet@utoronto.ca
}

Supplemental Information 


\section{Abbreviations:}

2D, two-dimensional; 3D, three-dimensional; $\mathrm{ACN}$, acetonitrile; $\mathrm{CNS}$, central nervous system; $\mathrm{DCM}$, dichloromethane; c-HA $\times \mathrm{MC}$, cleaved $\mathrm{HA} \times \mathrm{MC}$; DIC, N,N'-diisopropylcarbodiimide; DIPEA, N,N'-diisopropylethylamine; DMF, dimethylformamide; DMSO, dimethylsulfoxide; DMTMM， 4-(4,6-dimethoxy-1,3,5-triazin-2-yl)-4-methylmorpholinium chloride; DOSY, diffusion ordered spectroscopy; DS, degree of substitution; DTT, 1,4-dithiothreitol; GFP, green fluorescent protein; HA, hyaluronan; HAK, hyaluronan-ketone; HAMC, physically crosslinked hyaluronan/methylcellulose hydrogel; HA $\times \mathrm{MC}$, TEV enzyme-degradable hyaluronanmethylcellulose hydrogel; hPSCs, induced pluripotent stem cells; iPSCs, induced pluripotent stem cells; MC, methylcellulose; MCA, methylcellulose-aldehyde; $\mathrm{MC}-\mathrm{COOH}$, methylcellulosecarboxylic acid; MES, 2-(N-morpholino)ethanesulfonic acid; MW, molecular weight; NGD, neuro-glial differentiation; NMR, nuclear magnetic resonance; PBS, phosphate buffered saline; PEG, poly(ethylene glycol); PEG-OA 2 , PEG modified with oxyamine groups, TEV, tobacco etch virus; TEV-OA 2 , TEV protease degradable peptide modified with oxyamine groups; TNBS, 2,4,6trinitrobenzenesulfonic acid.

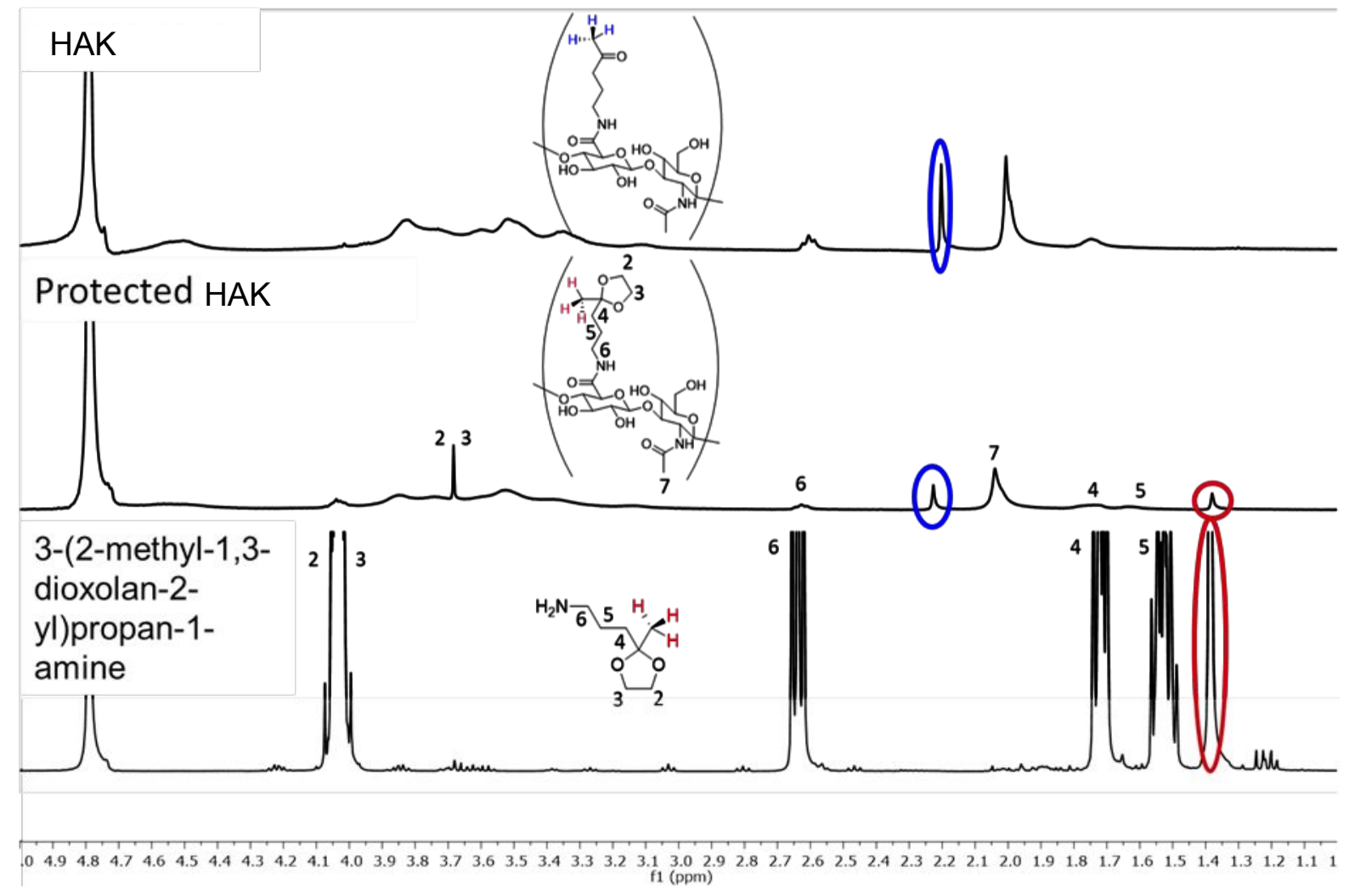

Figure S1. Stacked ${ }^{1} \mathrm{H}$ NMR spectra of HAK and its corresponding conjugated small molecule. 


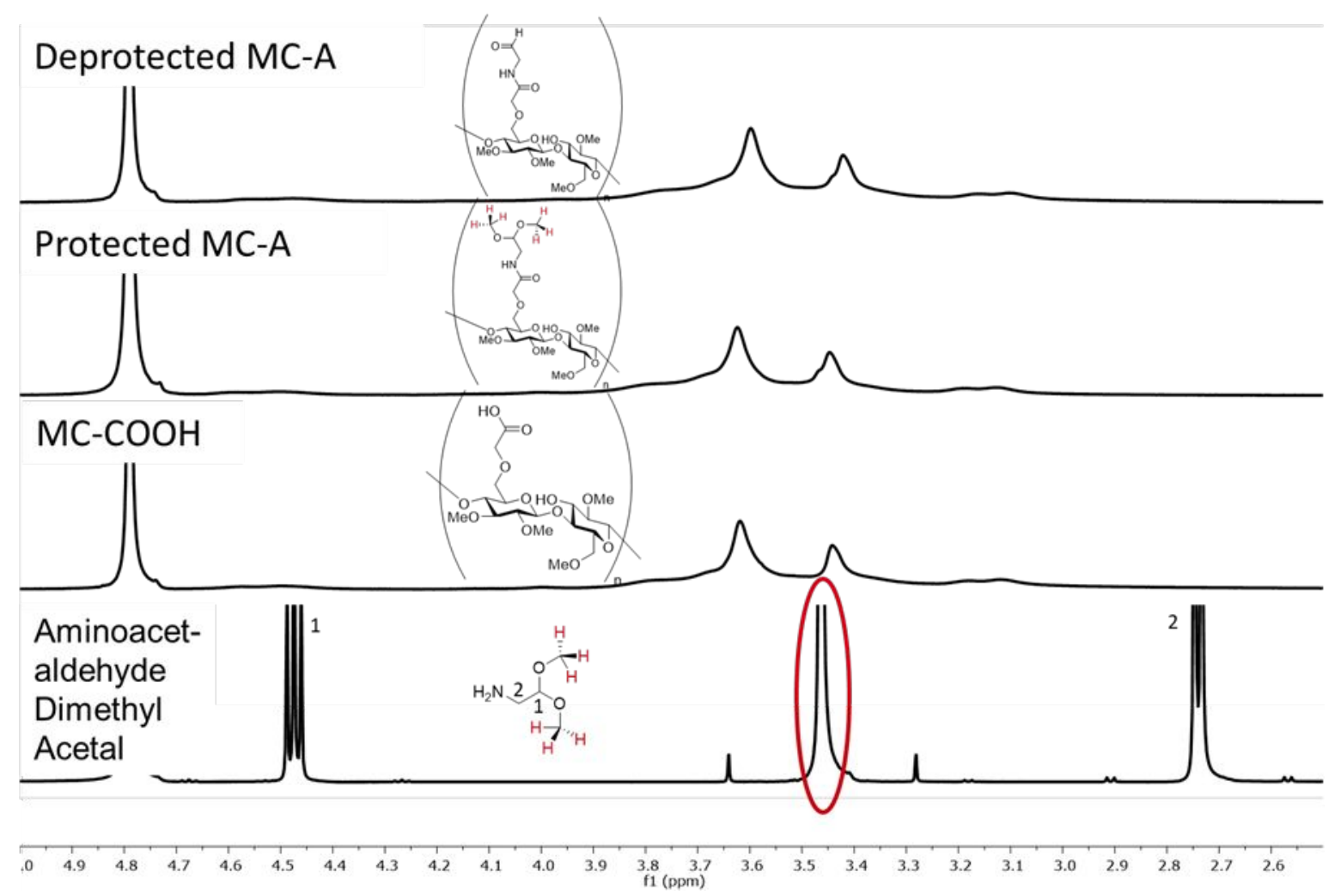

Figure S2. Stacked ${ }^{1} \mathrm{H}$ NMR spectra of MCA and its corresponding conjugated small molecule. As can be seen above, the protons on the acetal group were buried beneath the methoxy protons on the MC backbone, therefore MCA was reacted with t-butyl carbazate to quantify DS.

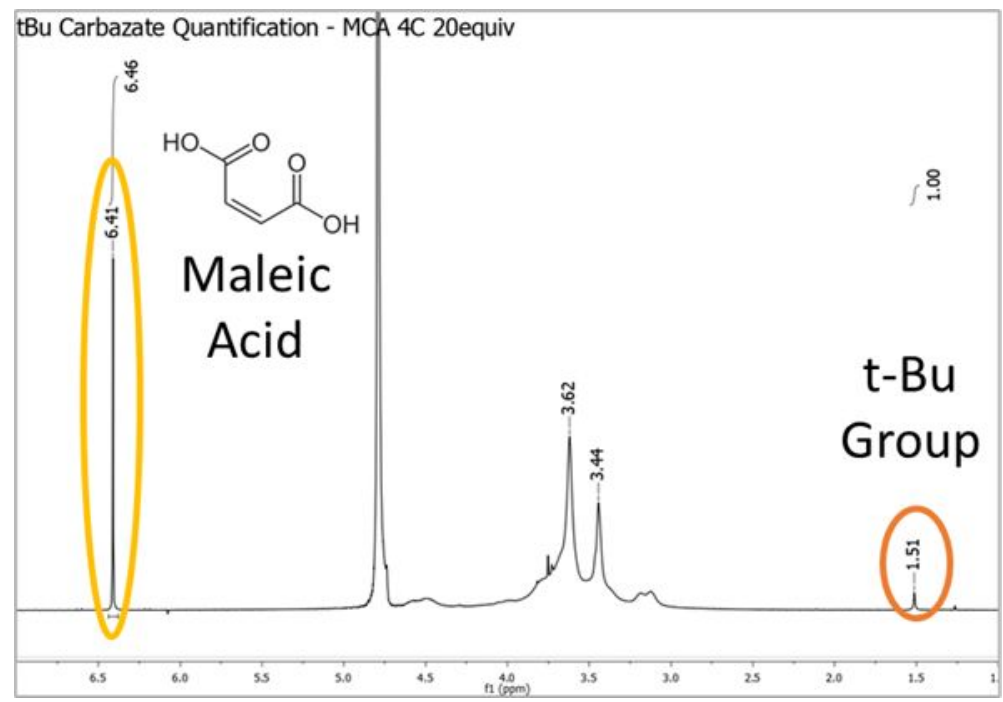

Figure S3. ${ }^{1} \mathrm{H}$ NMR spectrum of t-butyl carbazate modified MCA with maleic acid as an internal standard. 


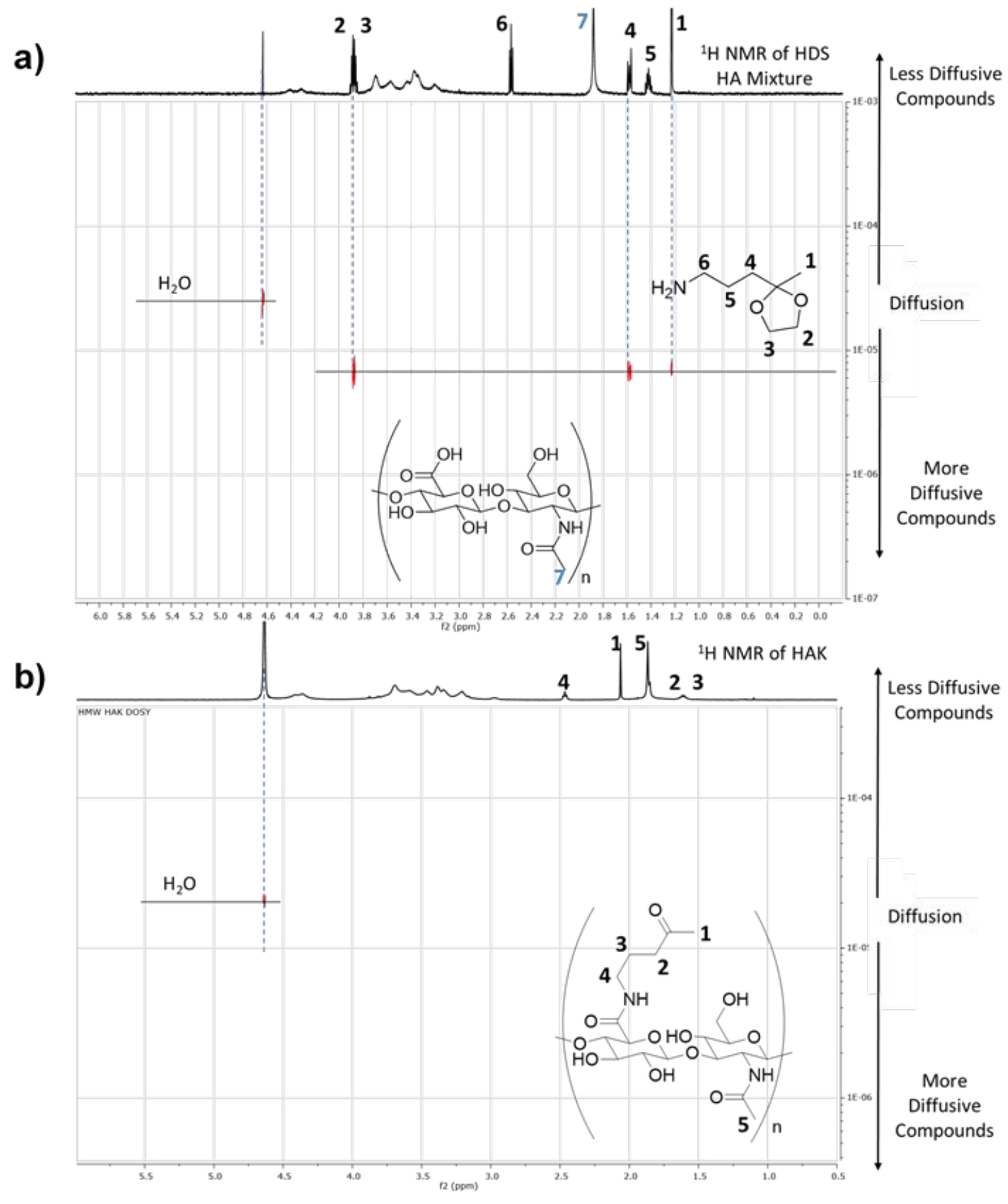

Figure S4. DOSY spectra of modified and unmodified HA. a) DOSY spectrum of HA mixed with the free small molecule 3-(2-methyl-1,3-dioxolan-2-yl)propan-1-amine. Independent diffusion of the small molecule from the polymer is observed, indicating no covalent bonding between HA and 3-(2-methyl-1,3-dioxolan-2-yl)propan-1-amine. b) DOSY spectrum of HAK. 3-(2-methyl-1,3dioxolan-2-yl)propan-1-amine does not diffuse independently from the polymer backbone, indicating that the two are covalently conjugated. 


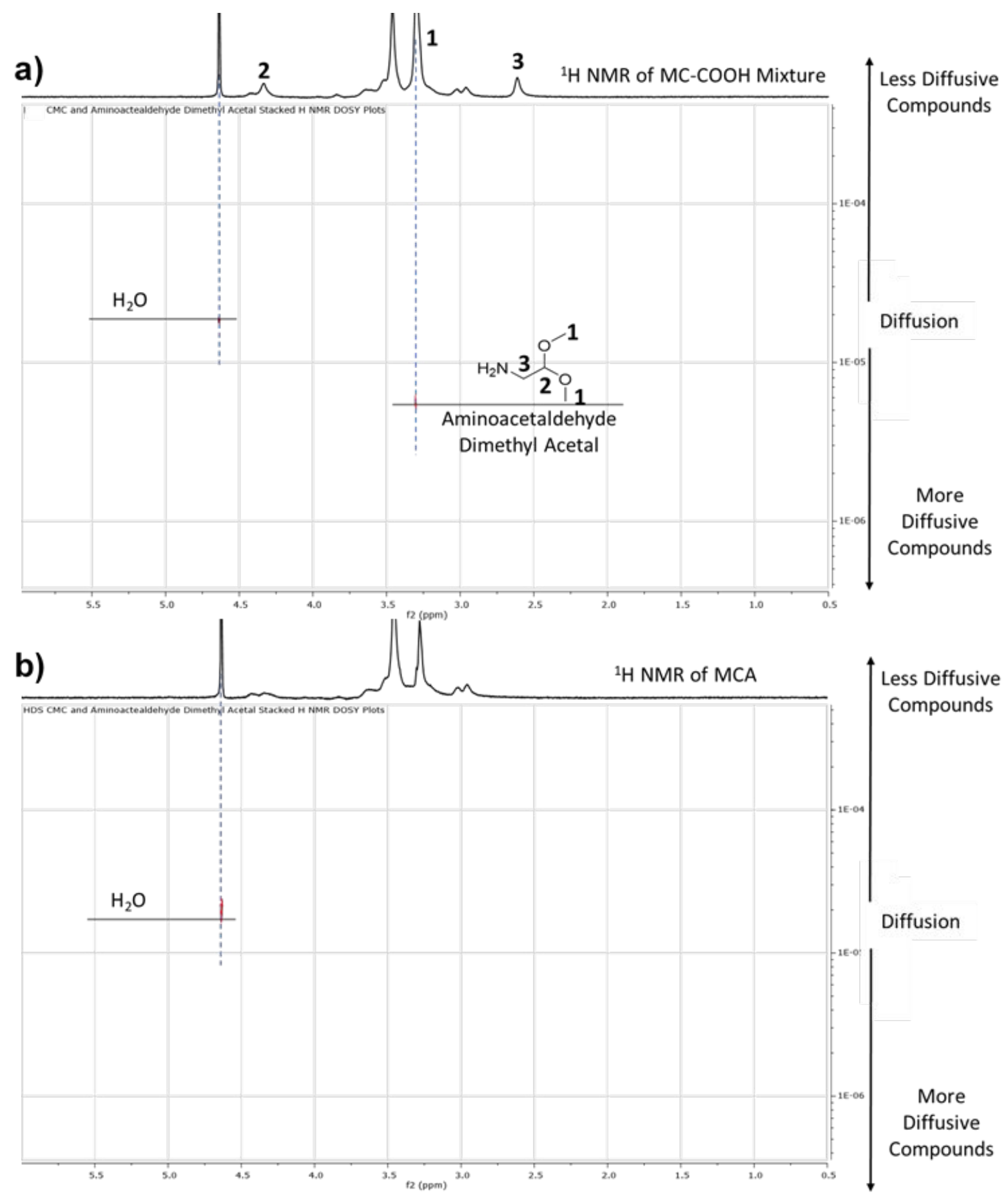

Figure S5. DOSY spectra of modified and unmodified MC. a) DOSY spectrum of MC-COOH mixed with the free small molecule aminoacetaldehyde dimethyl acetal. Independent diffusion of the small molecule from the polymer is observed, indicating no covalent bonding between MC$\mathrm{COOH}$ and aminoacetaldehyde dimethyl acetal. b) DOSY spectrum of MCA. Aminoacetaldehyde dimethyl acetal does not diffuse independently from the polymer backbone, indicating that the two are covalently conjugated. 


\section{H-S-K-A-G-E-N-L-Y-F-Q-S-G-A-K-S-K-S-OH}

Chemical Formula: $\mathrm{C}_{78} \mathrm{H}_{124} \mathrm{~N}_{22} \mathrm{O}_{27}$

Molecular Weight: 1801.96

$\begin{array}{lllll}\text { Sample Name } & \text { KS1-81 TEVX-Fmoc Protected,Alloc Deprotected-2 } & \text { Data File } & 190424 \text { 4543.d } & \text { Acq Method } \\ \text { DA Method } & \text { AIMS_Peptide.m } & \text { Instrument } & \text { Agilent } 6538 \text { UHD } & \text { Acq Date, Time } \\ \text { Comment } & \text { ESI+ } & & 4 / 24 / 201\end{array}$

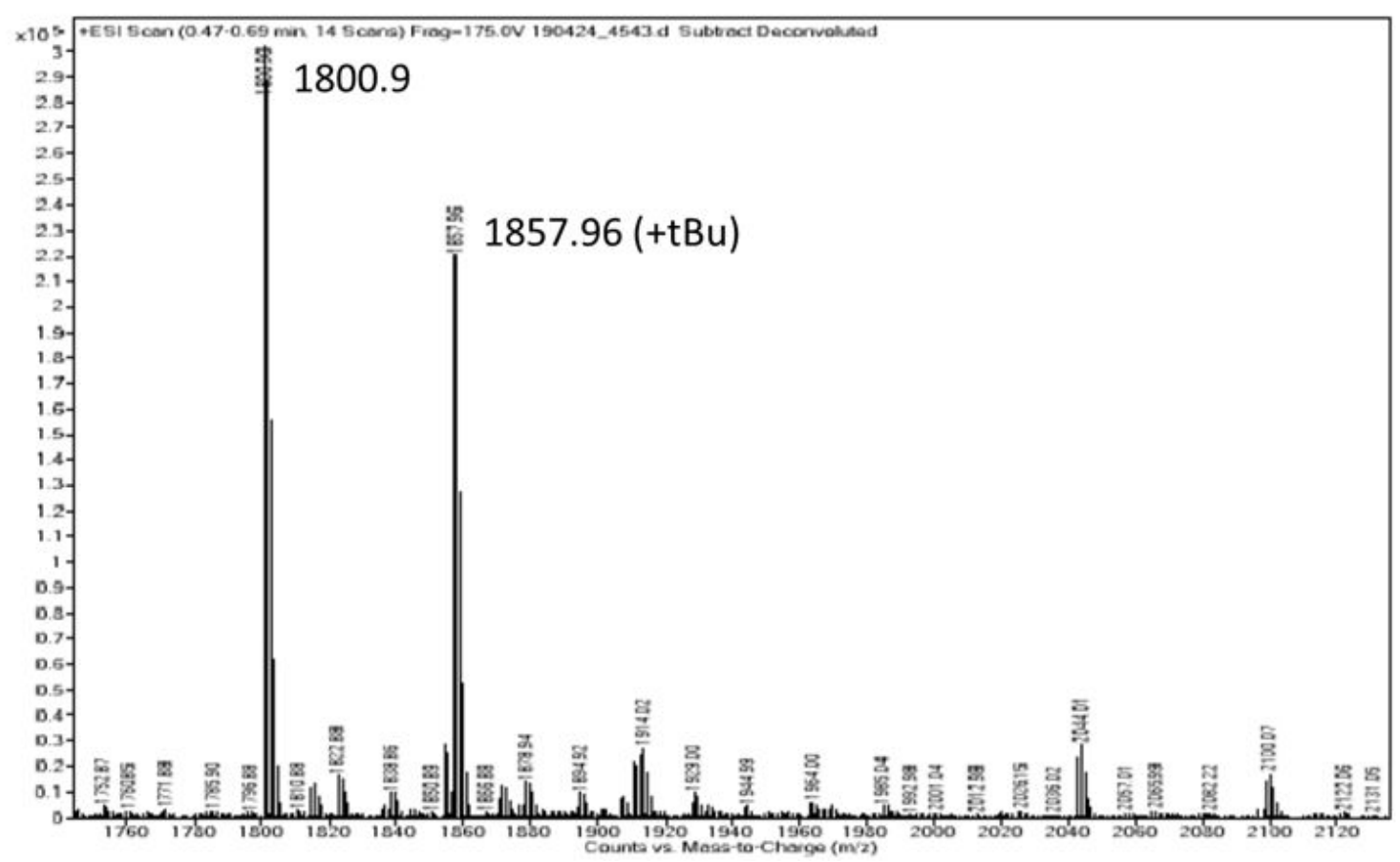

Figure S6. Mass spectrum of TEV peptide sequence before modification. 


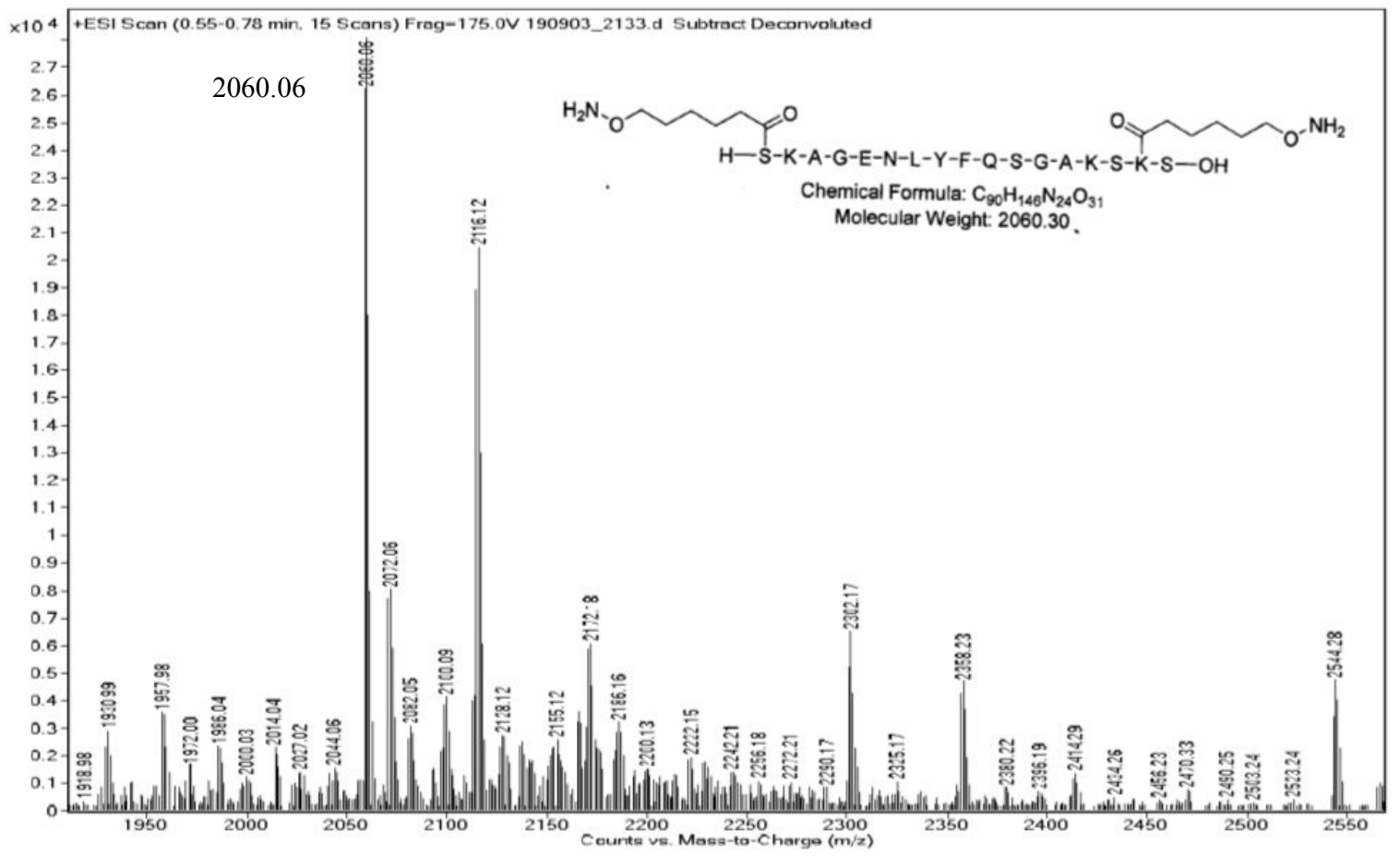

Figure S7. Mass spectrum of TEV-OA 2 prior to purification via $\mathrm{C} 18$ column chromatography. The main peak at $2060.06 \mathrm{~m} / \mathrm{z}$ corresponds to the expected mass of TEV-OA 2 . The peak at 2072.06 $\mathrm{m} / \mathrm{z}$ corresponds to a change in $+12.00 \mathrm{~m} / \mathrm{z}$ from the main peak and represents an unintended reaction between TEV-OA 2 and a formaldehyde impurity. The second largest peak at $2116.12 \mathrm{~m} / \mathrm{z}$ corresponds to a change in $+56.06 \mathrm{~m} / \mathrm{z}$ from the main peak and represents an unintended reaction between TEV-OA 2 and a tert-butyl cation from a cleaved amino acid protecting group. Other peaks at $2172.80 \mathrm{~m} / \mathrm{z}, 2302.17 \mathrm{~m} / \mathrm{z}$, and $2358.23 \mathrm{~m} / \mathrm{z}$ represent further reaction of TEV-OA $\mathrm{A}_{2}$ with tertbutyl groups, that being 2,4 , and 5 tert-butyl groups, respectively. 


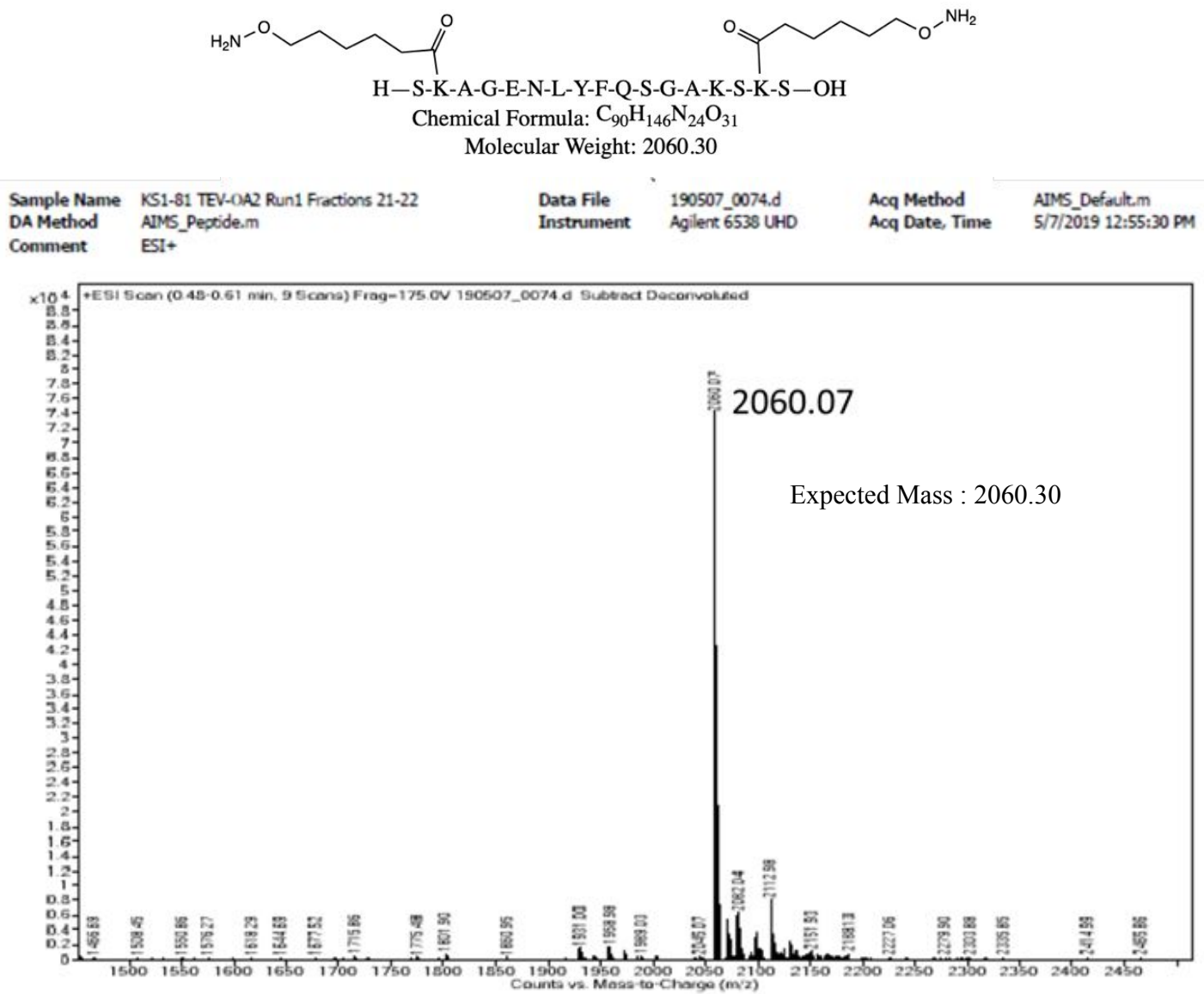

Figure S8. Mass spectrum of purified TEV-OA 2 . 


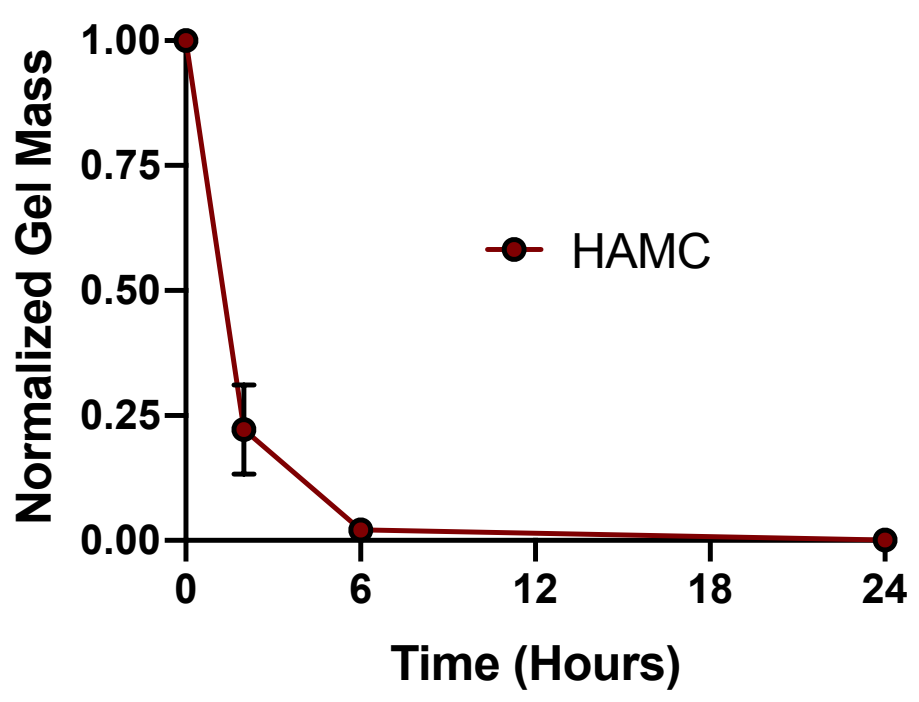

Figure S9. Stability study of $300 \mu \mathrm{L}$ HAMC. All hydrogels were prepared at 0.4:0.4 w/w of HA:MC. The HAMC gels dissolved completely by $6 \mathrm{~h}(\mathrm{n}=4$, mean \pm standard deviation). 

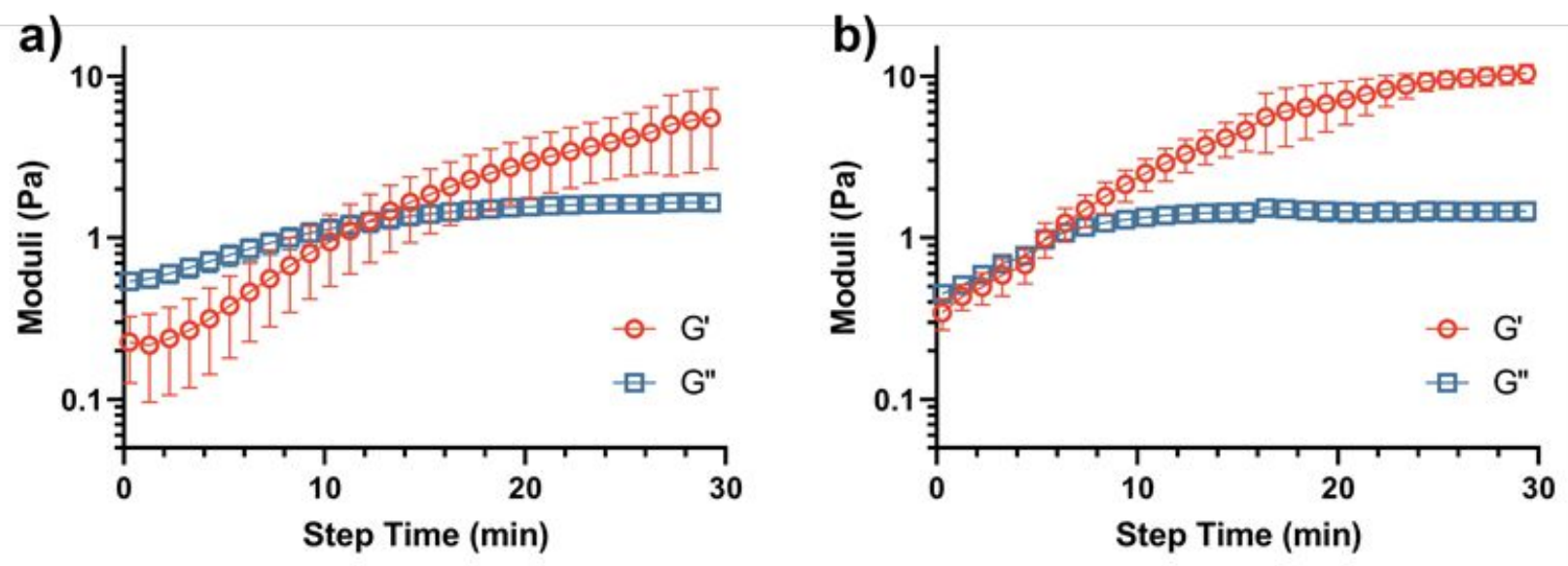

Figure S10. Gelation of 0.4:0.4 wt $\%$ HAK:MCA with $40 \%$ crosslink density as determined through rheology. a) Gelation time of $\mathrm{HA} \times \mathrm{MC}$ at $25^{\circ} \mathrm{C}$ was found to be $11.8 \pm 0.7$ minutes. b) Gelation time of $\mathrm{HA} \times \mathrm{MC}$ at $37^{\circ} \mathrm{C}$ was found to be $4.8 \pm 1.1$ minutes. Gelation time was determined by calculating linear equations for G' and G" near their point of interception and then calculating their intercept. Gelation time error was calculated through differential error analysis. $n=3$, mean \pm standard deviation.

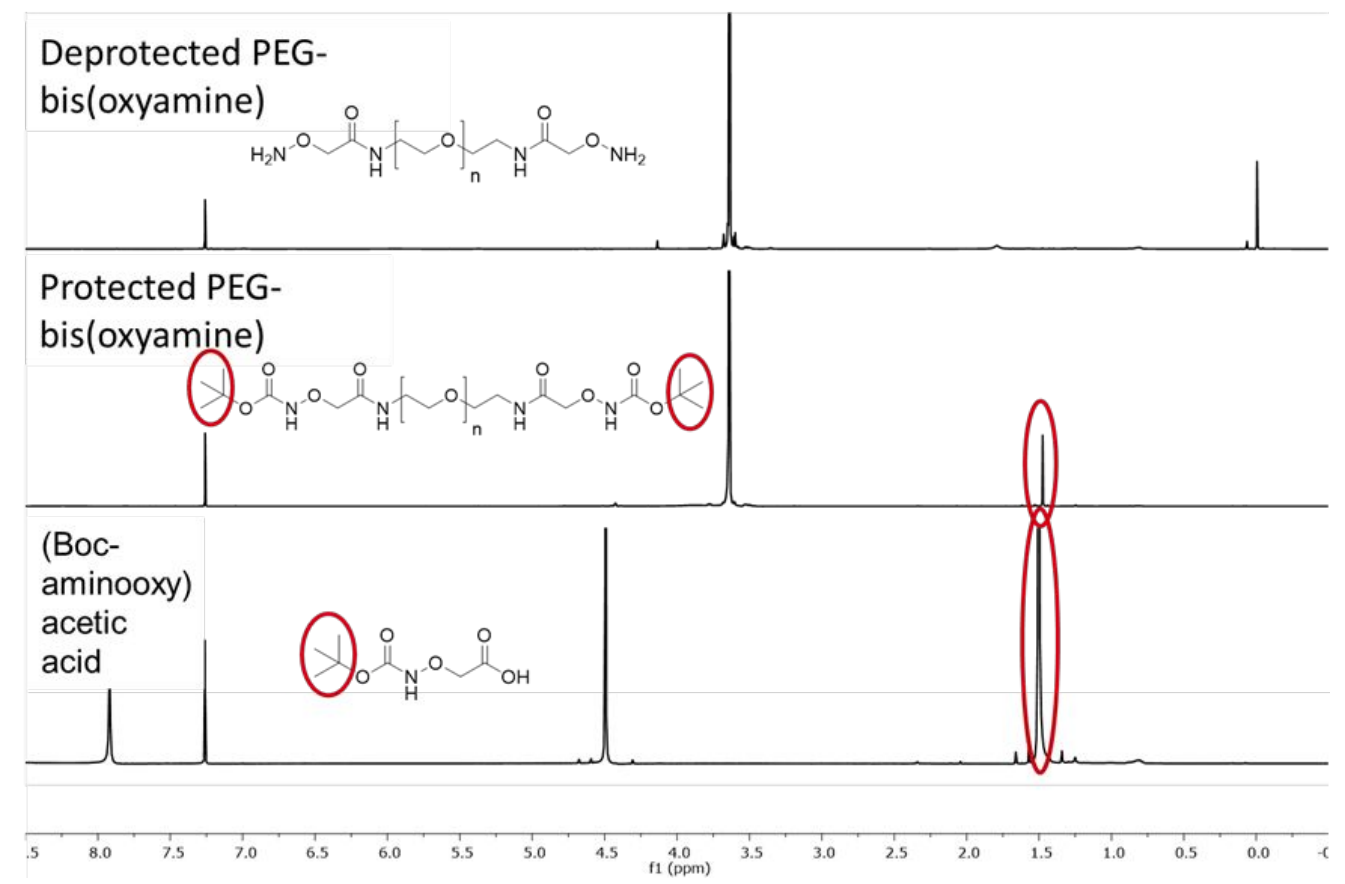

Figure S11. Stacked ${ }^{1} \mathrm{H}$ NMR spectra of PEG-OA 2 and its corresponding conjugated small molecule. 